due much more to the gauze drains than to any other factor. An analogy may be found in the postoperative acihesions in the female pelvis following prolonged drainage. The omission of drainage results in the omission of adhesions.

We have carried out this technic in consecutive operations only in the past three months, during which time we have operated on eleven patients, with one of whom only did we vary the technic by placing a piece of rubber dam down to the duct. In the ten cases, there was prompt recovery with a convalescence that was exceptionally smooth. In the one case, in which the rubber dam was used, an accumulation of purulent bile developed on the third or fourth day and was evacuated, after which recovery was prompt.

We began this practice following five years' experience in closing without drainage in our cholecystectomies during which, in a considerable series of cases, we have had no occasion to regret our action. As in the case of cholecystectomy, we do not feel that every common duct operation should be completed without drainage. It would not be safe in the presence of a virulent infection with severe inflammatory reaction present. It cannot easily be done when the duct is small. The frequency of the need of drainage is possibly illustrated by this series in which it was used once in eleven consecutive cases. A condition that occasionally requires gauze packing is oozing from the liver surface. This is unusual and did not occur in the series, though the gallbladder was removed in each case.

We offer these suggestions with a feeling that the one radical improvement in the technic of gall tract surgery is the omission of external drainage, and that a wider use of this method will demonstrate not only its feasibility, but also its comparative safety.

104 South Michigan Avenue.

\section{RENAL FLUOROSCOPY AT THE OPERATING TABLE*}

W. F. BRAASCH, M.D., AND R. D. CARMAN, M.D. ROCHESTER, MINN.

Surgical treatment of renal lithiasis is rendered unsatisfactory by complications as follows:

1. The difficulties of an exact interpretation of the roentgenogram.

2. The difficulty of locating the stone.

3 . Inability to find the stone.

4. The possibility of overlooking one or more stones when multiple stones are present.

5. The possibility of having fragments of stones broken off or loosely connected with the original stone.

It is true that a large number of renal stones are easily diagnosed and removed. This is particularly true of stones with a diameter of two or more centimeters, situated in the pelvis. When the stone is small and flat, however, or when it is deep in the calix, projecting into the cortex, palpation of the stone may be impossible, even when the kidney is brought out of the incision. The possibilities of error in diagnosis have been greatly reduced through the aid of cystoscopy and pyelography. Nevertheless, it may be quite impossible (1) to differentiate extrarenal

* From the Section on Urology and the Section on Roentgenology, Mayo Clinic. shadows which are obscured by the renal pelvis outlined in the pyelogram; (2) to identify small stones in the kidney that have not caused any pathologic changes in the outline of the renal pelvis, and (3) to recognize calcareous patches which are occasionally found in the kidney cortex and differentiate them from actual sione.

The preoperative localization of the shadow has been made fairly accurate (1) by interpretation of the size and shape of the shadow; (2) by the relation of the kidney to the shadow, and (3) by means of pyelography. Stones with a triangular or branched outline are almost always situated in the pelvis. If the outline of the kidney is definitely determined by the roentgenogram, the position of the stone may frequently be approximately inferred. Although pyelography will usually afford even more accurate data in the localization of the stone, it may also be inexact. It may be impossible to determine whether the stone is free in the upper calix, impacted in the end of the calix, or projecting partially or wholly into the cortex.

Nothing is more disconcerting than the inability to find a stone in the kidney when the various methods of clinical examination have definitely shown it to be there. In the presence of a hydronephrosis, particularly. when the dilatation is largely confined to the calices, a stone of fair size may be secreted in the bottom of a calix and defy discovery by the palpating finger. A stone lodged in the end of a calix which is shut off from the pelvis by inflammatory or cicatricial change may escape palpation. When the stone is in the cortex, the venous congestion of the kidney consequent to delivery may render its palpation exceedingly difficult. Needling, while occasionally of aid, is more often of doubtful value, and since it causes considerable damage to the kidney tissue, should be discouraged. Cortical incision, when extensive and multiple, is objectionable because of consequent destruction of renal tissue and the danger of subsequent hemorrhage.

It may be very difficult to determine, from the roent. genogram, the number of stones in a kidney. The shadow may appear to be single when in reality it represents two stones which either overlap or are closely approximated. What appears to be a singie branched stone may be made up of several distinct stones. The shadow may be misleading when it has a projecting branch which is seemingly explained by irregularity in the stone removed; a second small stone which actually caused the shadow may be easily overlooked. On the other hand, the shadow of an irregular stone may assume an outline and consistency suggestive of several stones. When only one stone is found at operation, the surgeon, after persistent search with more or less damage to the kidney, is still in doubt as to a remaining stone.

On the removal of branched stones, fragments are easily broken off, particularly when the ends are impacted in minor calices. Examination of the stone removed may show this, but more often it cannot be determined definitely. Rough stones that are extracted with difficulty may have a soft fragment wrenched off. Occasionally soft stones have a putty-like mass of crystals adjacent to them which may later form the nucleus of another stone.

Because of the difficulties in interpreting the roentgenograms and determining the number and location of stones, surgery for renal lithiasis is frequently an unsatisfactory procedure. Many so-called cases of recurrences of renal lithiasis are due to the fact that 
the stone or stones have not been completely removed at the time of operation. Whether or not stones or their fragments have been overlooked may often be determined by a roentgenogram made following convalescence from operation. This may give the desired information ; but if a shadow persists, the patient's condition will not be improved, and the surgeon is then faced with the awkward necessity of being obliged to advise either an immediate or a postponed operation. An immediate operation is rendered difficult by the patient's condition, by changed conditions of the perirenal tissues, the possibility of renal hemorrhage, and so forth. If the operation is postponed, the patient may neglect the condition until the kidney becomes seriously damaged.

Realizing the difficulties involved, it is apparent that a more accurate method of examination of the kidney at the time of operation is desirable. Unfortunately, the usual roentgenographic examination at the operating table is an awkward procedure and requires too much time. It would seem that if fluoroscopic examination when the kidney is brought out of the wound could be made practical, the various diffculties surrounding lithotomy would be readily overcome. Taking advantage of the recent improvement in fluoroscopic apparatus and simplification of roentgenographic machines, we have employed the apparatus described herewith.

\section{APPARATUS}

The apparatus used for making fluoroscopic observations of the kidney at the operating table is essentially the same as that used in the base and field hospitals of the army, but with certain minor changes which make it adaptable to civilian practice.

Such instruments (machines) consist of a transformer and autotransformer enclosed in a metal cabinet mounted on large casters for portability. To the cabinet is attached a tube stand with a horizontal arm having universal joints for supporting the tube. The tube is of the Coolidge radiator self-rectifying type, mounted in a lead glass shield.

The unit is small and compact, requiring less than $21 / 2$ square feet of floor space. It is of light weight, is portable, and has no moving parts which might cause noise and vibration. The current is turned on and off either by a hand or a floor switch. These portable units may be operated from the ordinary lamp socket without special wiring.

\section{TECHNIC}

As an essential preliminary, the roentgen-ray operator should wear goggles of smoked glass for about fifteen minutes before the observation is to be made in order that he may have the necessary dark-accommodation and retinal perception. The roentgen-ray unit should be placed as close to the operating table as possible, and the rays focused through a small diaphragm so that they will pass through the delivered kidney on the fluoroscopic screen. When the fluoroscopist is ready to make the roentgenoscopic examination, the hooded screen held in the left hand is placed over the eyes, and the goggles are removed. The current is turned on by means of a foot switch. In the right hand is held a sterilized metal-tipped rod 18 inches long with which the fluoroscopist accurately points to the stone shadow in the kidney. The exposure is-short, requiring little more than a flash. The various details can be easily arranged so that there is no interference with surgical asepsis.

\section{A CARDIOPLEURAL SUCCUSSION SIGN *}

\section{W. S. DUBOFF, M.D.}

EDGEWATER, COLO.

The object of this report is to describe and explain a most unusual-and to us, new-physical sign.

\section{REPORT OF CASE}

A man, aged 21, was admitted to the sanatorium, June 11, 1918, with a history of two years' illness. Because of repeated, large hemoptyses, in the presence of a unilateral left-sided tuberculosis, pneumothorax was induced, July 25. Moderate amounts of air (about 500 c.c.) were injected at intervals varying from a few days to four weeks, without reactions. November 1 , he developed a spontaneous pneumothorax with an effusion two weeks later, at first serous but rapidly becoming purulent. Because of pressure symptoms, he was aspirated, April 9, 1919, 900 c.c. of purulest fluid being removed and replaced by 600 c.c. of air. This was repeated, September 4.

June 20,1919, the patient complained of a constant noise in his chest which seemed worse at night and prevented sleep. Physical examination revealed a left-sided pyothorax to the clavicle, with a clear right lung and cardiac displacement to the right. The apex of the heart was neither visible nor palpable. The left border could not be percussed out because of flatness due to the effusion, while the right border was about $3 \mathrm{~cm}$. to the right of the right sternal margin. The heart sounds were regular, clear and without accentuations. A distinct, short splash could be heard at each systole over the entire precordium. The splash could be heard easily without a stethoscope by placing the ear before the open mouth of the patient. It was audible to the patient himself.

The explanation of this peculiar sign was not obvious roentgenologically until September 6. Fluoroscopy at this time disclosed a conical accumulation of fluid at the left base, apex down, with a fluid level above, pulsating distinctly with each heart beat. The lung was well collapsed, except at the base, which explained the conical appearance of the fluid. The heart was displaced moderately to the right. The right diaphragm was freely movable; the left diaphragm was immobile, the outer half being lost in the fluid shadow.

\section{COMMENT}

A splashing sound synchronous with the heart beat is described by the French as the "bruit de moulin" and is supposed to be characteristic of hydropneumopericardium. ${ }^{1}$ It occurred in nineteen out of thirty-eight cases collected by James, who also mentions it as occurring conceivably in hydropneumothorax, but cites no case. Speaking of pneumopericardium, Babcock" says, "Finally, the confounding of this disease with the presence of air and fluid in the pleural cavity is scarcely likely, if one will bear in mind that in pneumothorax the succussion sound is only obtained when the patient's body is agitated, while in the affection under discussion, the peculiar sound is present even when the patient is at rest."

This statement is not necessarily true. In our case pneumopericardium conld be definitely ruled out by the fluoroscopic examination alone, while the history and physical signs do not in the least suggest such a possibility. Obviously, our patient had a pericardialpleural adhesion which set the fluid in the pleural space suddenly in motion at each cardiac contraction.

* From the Sanatorium of the Jewish Consumptives' Relicf Society 1. James, W. B.: Tr. A. Am. Prys. 19:351-363, 1904. 2. Babcock, R. H.: Diseases of the Heart and Arterial Sysicm, Ed. 3, New York, D. Appleton \& Co.

Falling Birth Rate.-In the six months during March, 1919 the number of deaths in England and Wales exceeder the births by 126,000.-Medical Officer, Nov. 1, 1919. 